\title{
Observation of electron rings with imaging air Cherenkov telescopes
}

\author{
Haritha. Retnakaran ${ }^{1 *}$, Martin Tluczykont ${ }^{1}$, Dieter Horns ${ }^{1}$ \\ Institute for Experimental Physics, Universität Hamburg. \\ E-mail: haritha.retnakaran@uni-hamburg.de, \\ martin.tluczykont@physik.uni-hamburg • de, \\ dieter.hornsephysik.uni-hamburg.de
}

\begin{abstract}
The detection of muons in cosmic air showers with imaging air Cherenkov telescopes is an established method for the calibration of the detection efficiency of Cherenkov light of the entire optical system. Similar to muons, electrons passing the observation level near the telescope produce ring-like images. Images from electron rings can be potentially used to measure the electron energy and its energy-loss through ionization and Bremsstrahlung in the atmosphere. We use both, CORSIKA simulations as well as analytical calculations, to predict the shape of electron rings and the number of visible photons generated by electrons in the air shower. We investigate the opportunity to register electron rings with the current imaging air Cherenkov telescopes. It can be expected that the electron ring images are distinctly different from muon generated rings: since electrons below the critical energy continuously lose energy through ionization and suffer multiple elastic scattering, the rings are less sharp than the muon-induced rings. Our future plans will be to check the visibility of rings with the current and next generation imaging Cherenkov telescopes (e.g. HESS Phase II, MAGIC, TAIGA, CTA) by calculating an estimate for the rate of observable rings and the relevant energy range of cosmic-rays. Furthermore we will perform a search for electron rings in data from observations with low altitude cloud coverage. Finally, we will consider the possibilities to reconstruct the energy of electrons in the air shower using the observed electron rings.
\end{abstract}

36th International Cosmic Ray Conference -ICRC2019-

July 24th - August 1st, 2019

Madison, WI, U.S.A.

${ }^{*}$ Speaker. 


\section{Introduction}

Cherenkov radiation is emitted when a charged particle (such as an electron) passes through a dielectric medium faster than the phase velocity of light in that medium. When a particle moves with a velocity $\beta c$ in a medium with refractive index $n$, the angle $\theta_{c}$ of emission for Cherenkov radiation relative to the direction of the particle is given by:

$$
\cos \theta_{c}=\frac{1}{\beta n}
$$

which implies that there is a threshold value of $\beta_{\text {min }}=\frac{1}{n}$. The detection of electron rings requires that the electron reaches the observational level. The minimum energy required can be estimated using the longitudinal particle number of a photon initiated air shower. According to [1] and [2], the formula for the total number of electrons in a photon-initiated shower can be written as:

$$
N(t) \sim \frac{0.31}{\left(\beta_{0}\right)^{1 / 2}} \exp \left[t\left(1-\frac{3}{2} \ln s\right)\right]
$$

where $\beta_{0}=\ln \left(E_{0} / E_{c}\right)$ in which $E_{0}$ is the energy of the incoming photon and $E_{c}$ is the critical energy (in air, $E_{c}=80 \mathrm{MeV}$ ). The atmospheric depth $t$ is in units of radiation length $X_{0}$ of electrons; where $X_{0} \approx 37 \mathrm{~g}$ $\mathrm{cm}^{-2}$ in air. Other parameters such as the age parameter $s$ are defined in [1]. In Fig. 1, the total number of electrons in two photon induced showers of different starting energies over a range of atmospheric depth is shown. From this figure, one can see that the number of electrons at $t=20 \ldots .25$ (typical for observational sites) is sufficiently large to detect the electron rings. We leave a detailed calculation of the expected rates for future work.

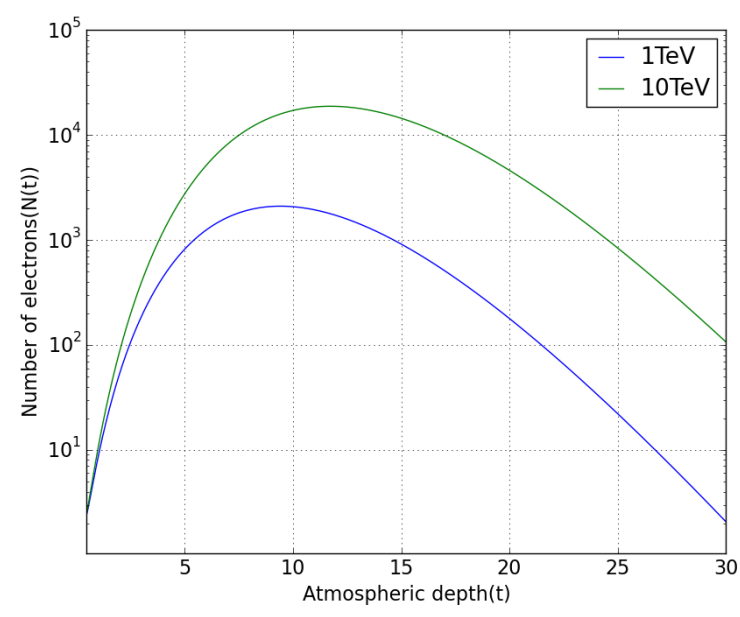

Figure 1: Number of electrons obtained from a photon-induced shower with primary energy $1 \mathrm{TeV}$ and $10 \mathrm{TeV}$ as a function of atmospheric depth $t$ in units of radiation length. The typical observation level of air Cherenkov telescopes ranges from $20 \ldots 25$.

\section{Energy-loss of charged particles}

When a charged particle passes through the atmosphere, it loses energy either by non-radiative process like ionization or radiative process like Bremsstrahlung. 


\subsection{Collision energy loss: Ionization}

Charged particles while passing through matter ionize or excite atoms on their path by colliding and transmitting energy to atomic electrons. The expression for the mean rate of energy loss is given by the Bethe-Bloch Equation:

$$
-\frac{d E}{d x}=K z^{2} \frac{Z}{A} \frac{\rho}{\beta^{2}}\left[\frac{1}{2} \ln \frac{2 m_{e} c^{2} \beta^{2} \gamma^{2} T_{\max }}{I^{2}}-\beta^{2}\right]
$$

where the value of constants are taken from the Particle Data Group [3]. The Bethe-Bloch equation can be used to analytically calculate the stopping power of particles (here electrons), see Fig. 2.

\subsection{Radiative energy loss: Bremsstrahlung}

When a charged particle is accelerated, it emits radiation and hence loses energy. In a sufficiently dense medium like the atmosphere, Bremsstrahlung dominates over competing loss mechanisms like Synchrotron and inverse Compton scattering. The energy loss per unit length due to Bremsstrahlung can be calculated as [1]:

$$
-\frac{d E}{d x}=\frac{E}{X_{0}} .
$$

In Fig. 2, we show the ionization losses together with Bremsstrahlung losses of an electron in air. Here we can define a critical energy $E_{c}$ at which $\left(\frac{d E}{d x}\right)_{\mathrm{rad}}=\left(\frac{d E}{d x}\right)_{\text {collision }}$. From Fig. 2, it can be seen that the critical energy is around $\beta \gamma \approx 160$, corresponding to $E_{c}=80 \mathrm{MeV}$.

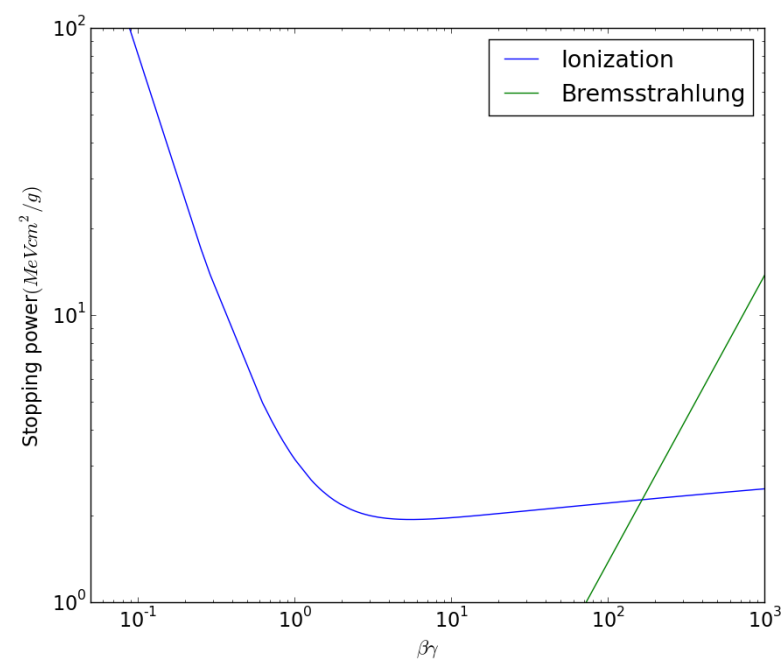

Figure 2: Energy loss of electrons as a function of their momentum.

\section{Tracing an isolated electron in the lower atmosphere}

The Lorentz factor of an electron injected at a height of $500 \mathrm{~m}$ above sea-level is calculated taking into account continuous energy losses including ionization and Bremsstrahlung. Subsequently, the Cherenkov emission angle and the total number of photons is calculated using a wavelength dependent model for the refractive index given by [4]. We use analytical calculations to study the electron rings produced in the image. With the help of equations 2.1 and 2.2, we calculate the Lorentz factor of these electrons. This has become possible by combining energy loss from ionization (2.1) and Bremsstrahlung (2.2) and substituting for $E=\gamma m_{e} c^{2}$ in both equations and then solving those first order differential equations in terms of $\gamma$ 
using different initial conditions $\gamma_{0}$. Fig. 3 shows the Lorentz factor of electrons starting at a height $500 \mathrm{~m}$ above ground level. Each of the curves are for electrons with various initial momenta. The horizontal line corresponding to $E_{c}$ shows the critical energy below which the ionization effect is dominant with respect to Bremsstrahlung. The dashed lines indicate the minimum Lorentz factor to emit Cherenkov light at the wavelengths $\lambda=300 \mathrm{~nm}$ and $\lambda=700 \mathrm{~nm}$.

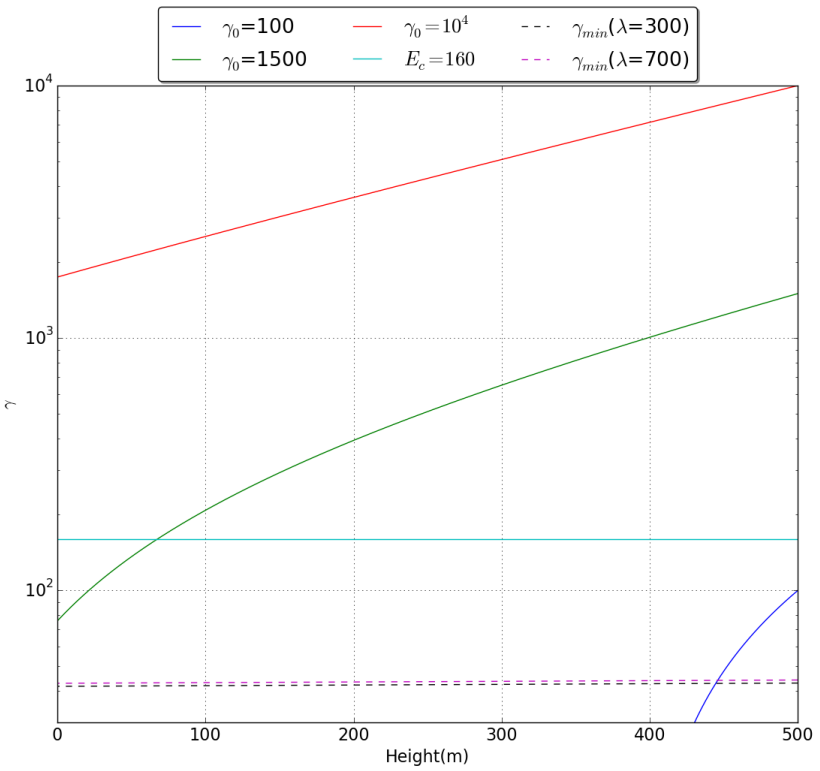

Figure 3: Lorentz factor of electrons for various initial conditions $\left(\gamma_{0}\right)$ as a function of the emission height, including ionization and Bremsstrahlung losses. The cyan line indicates the critical energy.

\section{Cherenkov light emission of an isolated electron}

\subsection{Angle of emission}

The emission angle of an electron propagating with an initial Lorentz factor $\gamma_{0}$ can be calculated using the Eqn. 1.1. The resulting angles as a function of height are shown in Fig. 4 for three values of $\gamma_{0}\left(10^{2}, 1500,10^{4}\right)$. 


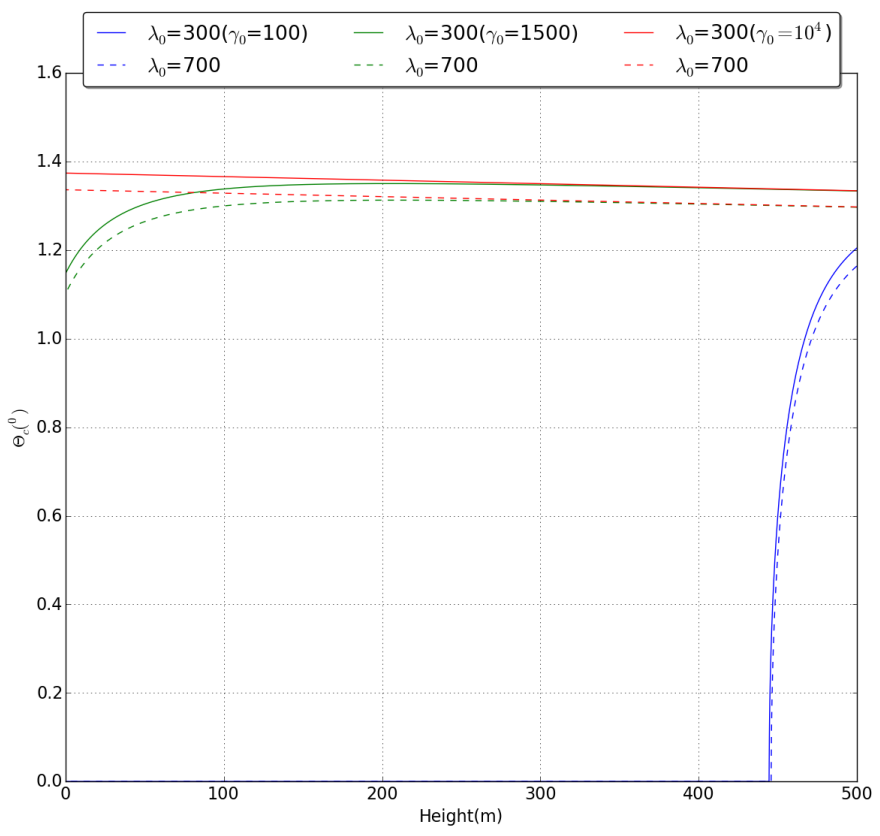

Figure 4: Angle of emission for electrons of three different energies as a function of the emission height.

\subsection{Radius of electron rings}

By considering the Cherenkov light cone of these electrons, we can calculate the expected cone radius on the ground. The angle of emission $\theta_{c}$, the emission height $h$, and the Cherenkov cone radius $r$ are related via $\tan \theta_{c}=r / h$. The resulting cone radius as a function of height above ground is shown in Fig.5 (for $\left.\gamma_{0}=10^{2}, 1500,10^{4}\right)$.

\subsection{Number of photons}

When a particle of charge $z e$ ( $z=1$ for electron) passes through a medium of refractive index $n$ with a velocity $\beta c$, the number of photons emitted per unit path length of that particle and per unit energy interval of the photons can be calculated using the following equation [3]:

$$
\frac{d^{2} N}{d x d \lambda}=\frac{2 \pi \alpha z^{2}}{\lambda^{2}}\left(1-\frac{1}{\beta^{2}(x) n^{2}(x, \lambda)}\right)=\frac{2 \pi \alpha z^{2}}{\lambda^{2}} \sin ^{2} \theta_{c}
$$

where $\alpha$ is the fine structure constant and $\lambda$ is the wavelength of the light being emitted. Equation 4.1 can be used to calculate the number of photons emitted per path length $\frac{d N}{d x}$ by integrating over the wavelength $\lambda$. The result is shown in Fig. 6 (for $\gamma_{0}=100,1500,10^{4}$ ).

Photons are emitted in a cone with infinitesimal thickness. We need to consider the dependence on the solid angle $\Omega$ viewed by an imaging telescope. In order to include the solid angle $\Omega$ assuming uniform emission over $\phi$, we write equation 4.1 in such a way that;

$$
\int \frac{d N}{d \Omega d x d \lambda} d \Omega=\frac{d N}{d x d \lambda}
$$

where $d \Omega=d \phi \sin \theta d \theta$. In order to fullfil equation 4.2, we define:

$$
\frac{d N}{d \Omega d x d \lambda}=\frac{\alpha z^{2}}{\lambda^{2} \sin \theta} \sin ^{2} \theta_{c} \delta\left(\theta-\theta_{c}(x)\right) .
$$




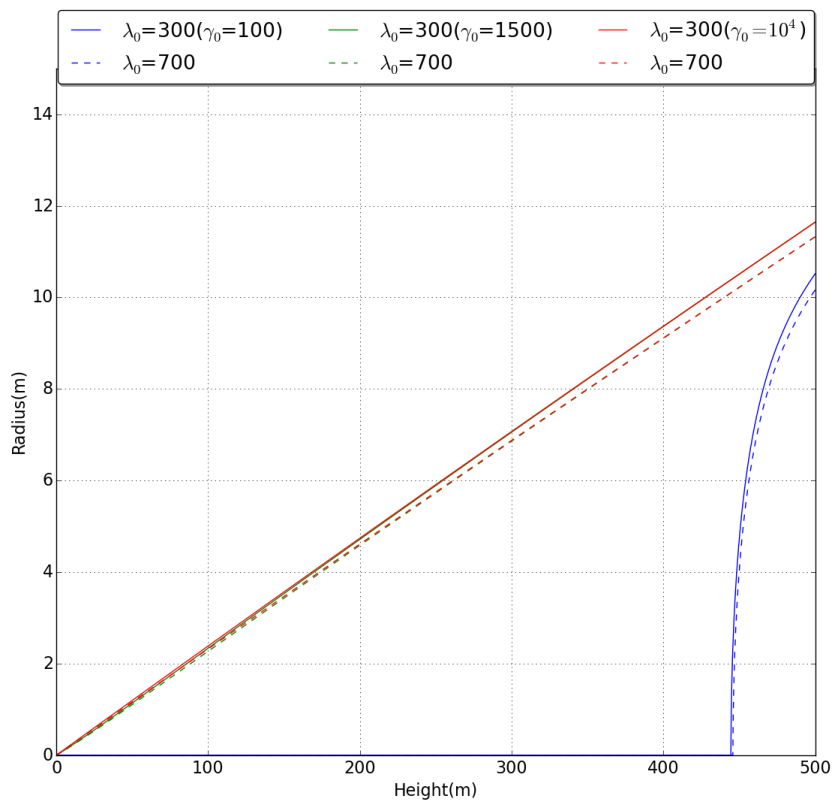

Figure 5: Cone radius for electrons of three different energies as a function of the emission height.

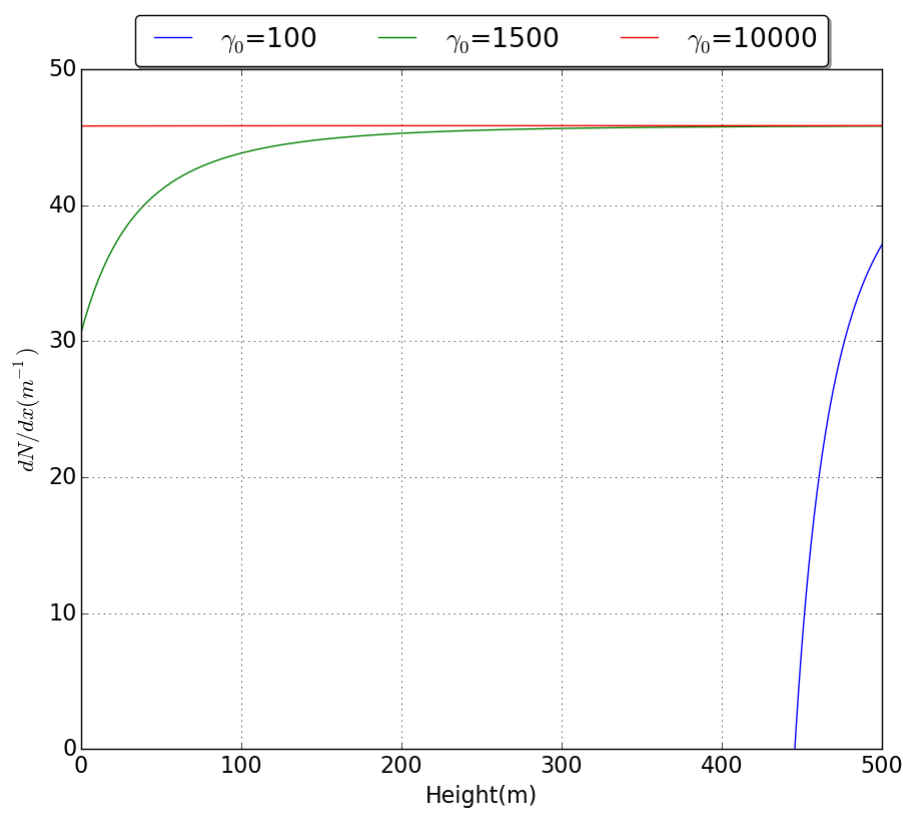

Figure 6: Number of photons emitted per path length for electrons of three different energies as a function of the emission height.

Carrying out the integration in equation 4.2 using equation 4.3 , we obtain per construction equation 4.1. 
Then equation 4.3 can be integrated over the path length ${ }^{1}$ :

$$
\frac{d N}{d \lambda d \Omega}=\frac{\alpha z^{2}}{\lambda^{2}} \int d x \sin \theta_{c} \delta\left(\theta-\theta_{c}(x)\right)
$$

using the properties of the $\delta$-distribution, we can carry out the integration and obtain:

$$
\frac{d N}{d \lambda d \Omega}=\frac{\alpha z^{2}}{\lambda^{2}} \frac{\sin \theta\left(x_{0}\right)}{\left|\frac{-d \theta\left(x_{0}\right)}{d x}\right|_{x=x_{0}}} .
$$

Using the number of photons per solid angle $\frac{d N}{d \Omega}$, we calculate the number of photons per typical pixel size of $1.33 \mathrm{mrad}$ and use it to calculate the rings analytically (see Fig.7). For the purpose of visualization, we choose to display the resulting image in $u$-v coordinates where $u=\sin \theta \cos \phi$ and $v=\sin \theta \sin \phi$.

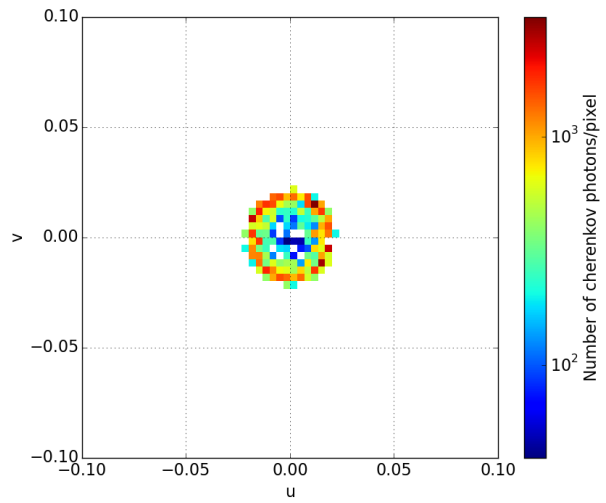

(a) $\gamma_{0}=10^{2}$

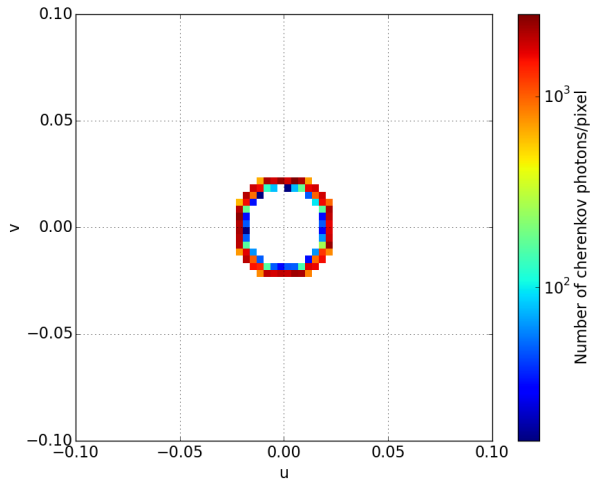

(b) $\gamma_{0}=1500$

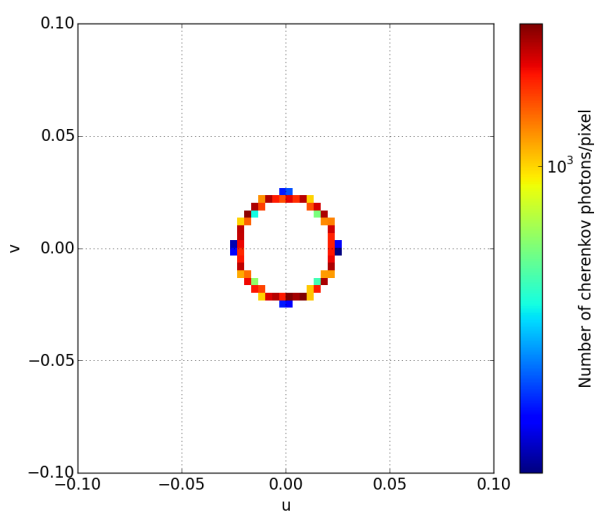

(c) $\gamma_{0}=10^{4}$

Figure 7: 2D histogram showing the $u v$ images of the electron rings with different initial energy

\footnotetext{
${ }^{1}$ Angle of emission $\theta_{c}$ is a function of wavelength $\lambda$ and height $x$ which makes $\delta$ function as two variable function (both $\lambda$ and $x$ ). For simplicity in this calculation, wavelength is fixed to $\lambda=500 \mathrm{~nm}$.
} 


\subsection{CORSIKA air shower simulation}

CORSIKA (version 7.6400) is a program used to simulate air showers initiated by high energy cosmicray particles[5]. Within CORSIKA, we calculated the emission from an isolated electron close to the detection plane, confirming our analytical result. Additionally, we consider the emission of electrons from an air shower with an initial energy of $10 \mathrm{TeV}$. The CORSIKA output contains information on the Cherenkov photons on the detector plane such as the $u v w$ coordinates, time of arrival, height of production and $x y$ coordinates. We create a $u v$ image from the photons which are within a radius of $5 \mathrm{~m}$ from the shower axis(see Fig 8). In this simulation, we have applied an energy cut (ELCUT(3) for electrons: see [5]) of $20 \mathrm{MeV}$ (well below $E_{c}$ ) which specifies the minimum energy up to which the electrons are tracked in CORSIKA. At an altitude of $110 \mathrm{~m}$ above the sea level, a total of 54 electrons/positrons pass through the detector plane. The resulting image includes several ring-like features with different radii as well as ring segments. Given the treatment of Molière scattering in CORSIKA, it appears feasible that multiple rings relate to one individual electron. Further studies are needed to disentangle the different effects included in the simulation.

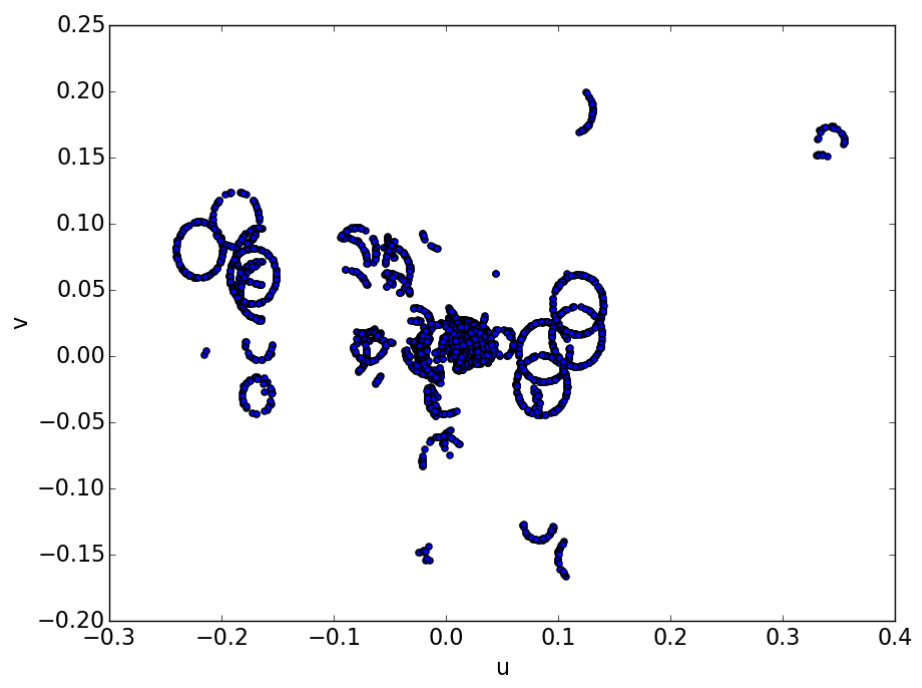

Figure 8: $u v$ image showing electron rings obtained from a $\gamma$-ray shower of $10 \mathrm{TeV}$

\section{Summary}

Cherenkov emission from electrons leads to the formation of rings of different radii. These rings can be used to determine the starting energy of the electron and also to study their behavior by the time they reach the ground. If we consider an electron with a particular starting energy, the resulting rings on the ground will be of slightly different radii with photons of different wavelength as can be seen in Fig 4. The sharpness of the rings increases with the energy of the secondary electrons.

Electrons will also experience multiple scattering. However we haven't considered the effect of multiple scattering in the analytical calculations which will have a substantial effect on both the energy loss (straggling) as well as the smearing out of ring features. We will consider the effect of Cherenkov light generated by low-energy electrons to experiments like MAGIC, TAIGA and CTA and compare with real data. We will also perform a search for electron rings in data from observations with low altitude cloud coverage which can hopefully give a possibility to reconstruct the energy of electrons in the shower. 


\section{References}

[1] Gaisser, Thomas K. and Engel, Ralph and Resconi, Elisa, Cosmic Rays and Particle Physics, Cambridge University Press, Cambridge 2016

[2] Greisen, K., Prog. Cosmic Ray Physics, Volume3 (1956) 1

[3] Patrignani, C. and others, Review of Particle Physics, Particle Data Group, Chin. Phys., Volume C40 2016

[4] Philip E. Ciddor, Refractive index of air: new equations for the visible and near infrared, Appl. Opt. 35, 1566-1573 (1996)

[5] D.Heck et al., Report FZKA 6019 (1998), Forschungszentrum Karlsruhe; http://wwwik.fzk.de/corsika/physics-description/corsika-phys.html 